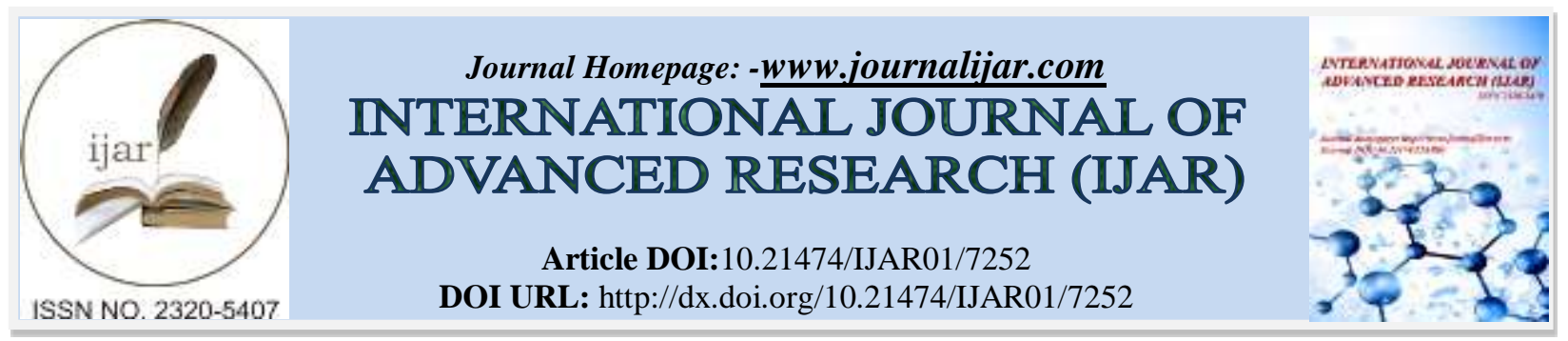

RESEARCH ARTICLE

\title{
NANO- HYDROXYAPATITE (NANO-HAP) REINFORCED GLASS IONOMER CEMENT:- AN ALTERNATIVE RESTORATIVE MATERIAL.
}

\section{Dr. Rajat Gupta ${ }^{1}$, Dr. Manvi Srivastava ${ }^{2}$ and Dr. Updesh Masih ${ }^{3}$.}

1. Post graduate Student, Department of Pedodontics and Preventive Dentistry, K. D. Dental College and Hospital, Mathura.

2. Senior Lecturer, Department of Pedodontics and Preventive Dentistry, K. D. Dental College and Hospital, Mathura.

3. Professor and Head, Department of Pedodontics and Preventive Dentistry, K. D. Dental College and Hospital, Mathura.

\section{Manuscript Info}

(.........................

Manuscript History

Received: 10 April 2018

Published: June 2018

Keywords:-

Bond strength, Micro hardness, Nano

Hap GIC, Zirconomer.
Final Accepted: 12 May 2018

\section{Abstract}

Context: The lack of evidence regarding the best available material for restoring occlusal-proximal cavities in teeth leads to the development of new restorative material, with nanoparticles, in order to enhance mechanical properties, resulting in increased restoration longevity.

Aim: To evaluate and compare the microhardness and shear bond strength of Nano Hap incorporated GIC and zirconomer with composite.

Materials and Method: Thirty permanent molars were selected and assigned into three groups according to the restorative materials used. The restorative material build up over the dentin surface was done All the specimens were stored in distilled water for $24 \mathrm{hrs}$ before shear bond strength testing. For micro hardness evaluation, ten blocks of each restorative material were made according to the groups assigned. All these specimens were stored in distilled water for $24 \mathrm{hrs}$ before microhardness testing.

Results: Nano- HAp GIC shows the highest Microhardness (112.79 VHN) followed by Composite (67.30) \& Zirconomer (47.96) whereas the shear bond strength of Composite (53.37 MPa) was found to be the highest when compared to HAp (3.98) \& Zirconomer (5.09).

Conclusion: Highest shear bond strength values were seen in composites followed by Zirconomer and Nano-hydroxyapitite (GIC). Nano-HAp showed the highest mean micro hardness values when compared to Zirconomer and composites.

Copy Right, IJAR, 2018,. All rights reserved.

\section{Introduction:-}

Restoring carious teeth is one of the major treatment needs of young children. Wide use of Glass-ionomer cements (GICs) in the restorative dentistry is attributed to their unique properties such as direct bonding to tooth structure, anti - cariogenic action due to release of fluoride and biocompatibility with pulp tissue. ${ }^{1}$ 
Despite of its wide applications \& various advantages, the main limitation of the glass ionomer cements is their relative lack of strength and low resistance to abrasion and wear. Conventional GICs have various other drawbacks too such as low shear bond strength (3-7 MPa), low flexural strength and high modulus of elasticity due to which these cements are very brittle and are prone to bulk fracture like limited strength, and microleakage. Microleakage is seen at the restoration - dentin interface resulting in postoperative senstivity, chronic hypersensitivity, secondary caries and pulpal pathosis. ${ }^{2}$

To overcome theses drawbacks, every time some new GIC formulations are introduced in the market by the manufacturers. Hence, it is very important to know the physical and mechanical properties of each product before selecting these new GICs as restorative materials. ${ }^{3}$ GICs with modified formulations marketed till date are Resinmodified GIC, compomers, carbomers, GICs with bioactive glass, Zirconomer and Nano - hydroxyapatite reinforced GIC.

Zirconomer or white amalgam has been newly introduced in the dentistry. Zirconomer is the high strength restorative material which has been reinforced with ceramic and zirconia fillers. Zirconia fillers provide good mechanical strength and dimensional stability. ${ }^{4}$ Hydroxyapatite (HA), the main mineral component of the tooth structure and bone, is a bioceramic containing calcium and phosphorus. The HA particles were added to glassionomer powder due to their biocompatibility and similar composition to apatite in human dental and skeletal systems. Several studies reported improvements in mechanical properties of these materials such as diameter, tensile strength, fracture toughness, bonding and compressive strength compared to conventional glass-ionomers Hydroxyapatite (HAp, Ca10 [PO4] $6[\mathrm{OH}]$ 2) is a typical biomineral that is abundant in organisms. It can be used as bone scaffolds and luminescence materials. 5,6 Thus, the present study was conducted with the aims to evaluate the shear bond strength and microhardness of conventional Glass Ionomer Cement (GIC) incorporated with Nanohydroxyapatite (Nano-HAp), Zirconomer and Composite and to compare the shear bond strength and microhardness of conventional Glass Ionomer Cement (GIC) incorporated with Nano -Hydroxyapatite (Nano-HAp) and Zirconomer with Composite.

\section{Materials and Method:-}

Ethical committee clearance was obtained before the beginning of the study. Thirty sound human permanent molars that had been extracted due to periodontal problems were collected and thoroughly washed in the running water immediately to remove any blood and adherent tissue. The teeth with cracks or fractures, malformations, carious lesions, restorations or erosions were excluded from the study. Scaling was done and the teeth were stored in distilled water at room temperature.

For the evaluation of the shear bond strength of the materials, the roots of all 30 selected teeth were separated from their crowns at the cemento- enamel junction using a diamond disc. A flat dentin surface was obtained on samples by removing the enamel over the occlusal portion with the help of high - speed carbide bur. The surfaces were embedded in the poly vinyl chloride moulds of $2.0 \mathrm{cms}$ in diameter, by using self cure acrylic resin (DPI, India) leaving the dentinal surface uncovered by the resin. These 30 samples were randomly divided into three groups depending upon the type of restorative material used i.e., Group A (composite), B (8\% w/w Nano HAp GIC) and C (Zirconomer). The restorative material build up over the dentin surface was done using a customized split metal casing of dimensions $6 \mathrm{~mm}$ diameter and $3 \mathrm{~mm}$ height, which was stabilized using a micropore tape. All the specimens were stored in distilled water for $24 \mathrm{hrs}$ before shear bond strength testing. The prepared samples were then subjected to shear bond strength evaluation using Instron Universal Testing Machine (Lloyd instruments Ltd., UK). A knife edge shearing rod and a crosshead speed of $1 \mathrm{~mm}$ per min were used.

For evaluation of micro hardness of the test materials, the specimens were prepared using a cylindrical metal mould with a diameter of $6 \mathrm{~mm}$ and $3 \mathrm{~mm}$ height were made and embedded into poly vinyl chloride moulds. Ten blocks of each restorative material were made according to the groups assigned.vAll these specimens were stored in distilled water for $24 \mathrm{hrs}$ before microhardness testing. The samples were subjected to micro hardness evaluation using MVK - H1 hardness testing machine (Mitueoyo, USA). Vicker's micro hardness measurements were made by applying a $300-\mathrm{g}$ load was applied for $15 \mathrm{~s}$.

The data thus obtained was subjected to statistical analysis which was performed using ANOVA Test, Post Hoc Tests for multiple comparisons using Tukey's Test. The data were analyzed using statistical package for social sciences version (SPSS) 17.0 for Windows. The level of statistical significance was set at $95 \%(P=0.05)$. 


\section{Results:-}

According to the Bar diagram 1, Nano- HAp GIC shows the highest Microhardness (112.79 VHN) followed by Composite $(67.30 \mathrm{VHN})$ \& Zirconomer (47.96 VHN) whereas the shear bond strength of Composite (53.37 MPa) was found to be the highest when compared to HAp (3.98 MPa) \& Zirconomer (5.09 MPa).

The intergroup comparison of shear bond strength values revealed that the $\mathrm{p}$ values were found to be significant when Group A was compared with Group B and C whereas no significant difference was found between Group B and Group C. (Table 1)

The intergroup comparison of microhardness values revealed that the $\mathrm{p}$ values were found to be significant among all the groups. (Table 2)

\section{Discussion:-}

There is a constant search for the materials that ensures adhesion to the tooth structure in order to minimize the failure of the restorations. Various restorative materials have been used since years to preserve the lost tooth structure and maintain form, function and aesthetics. The last few decades have witnessed efforts increasingly directed towards the discovery and development of newer dental materials and the modification of the older materials with the addition of various materials which were proven to be beneficial. GIC is known for its ability to chemically bond to the tooth structure, so it is of great concern that modification of the conventional GIC should not interfere with the physical properties and bonding ability of the original material. Two such modifications made were by incorporation of hydroxyapatite and zirconia fillers to the glass ionomer cements.

The incorporation of HAp can result in enhancing the strength of the matrix of GICs and resulting in better bonding between the glass core and glass matrix. The apatite formation of HAp in combination with the release of ions from glass ionomer can improve the mechanical properties of glass cements. Therefore, in the present study the effect of HAp on microhardness and shear bond strength, as two of the most important mechanical properties of the material was investigated. Nano HAp was chosen in this study because hardness value of HAp is similar to that of natural teeth and also nano-particles of HAp are more similar to the mineral phase of tooth structure than micro-particles as far as crystal size is concerned. ${ }^{7}$

Incorporation of zirconia into dental ceramics doubled their hardness; therefore, incorporation of zirconia into restorative glass-ionomer can enhance its microhardness. Sharafeddin F $(2017)^{2}$ found that the microhardness of Zirconomer was higher in comparison with conventional glass-ionomer whereas in the present study, the micro hardness of zirconomer was found to be least when compared to composite and nano - HAp GIC.

Kenji Arita concluded that the highest increase in the flexural strength of the GIC cement was achieved with the addition of $8 \%$ HAp granules in their study. ${ }^{8}$ Hence, same concentration of hydroxyapatite was used in the present study to check the influence on the shear bond strength and micro hardness of GIC.

One of the primary objectives of adhesion is to achieve a strong, durable and predictable union between restorative material and tooth structure. Bonding improves retention and stabilization of a restoration without excessive removal of sound tooth structure. Adhesive restorations are better able to transmit and distribute functional stresses across the bonded interface thereby, reinforcing weakened tooth tissue. The present study showed that the shear bond strength of nano-HAp incorporated GIC had very significantly lower values than composite. The values obtained for nano-HAp incorporated GIC and zirconomer were $3.98 \pm 0.23 \mathrm{MPa}$ and $5.09 \pm 0.34 \mathrm{MPa}$, respectively. Similar results were found in the study conducted by Choudhary $\mathrm{K}$ (2015). ${ }^{9}$ The mean shear bond strength values obtained for nano-HAp incorporated GIC ( $3.98 \pm 0.23 \mathrm{MPa})$ was lower to the value obtained from study by Moshaverinia et al. $^{10}$

In this study, intra-oral variables such as normal masticatory stress, moisture, intra-pulpal pressure, and operator inconsistencies were not taken into consideration. Therefore, further in vivo studies are necessary to test the long-term stability of these materials. 


\section{Conclusion:-}

Within the limitations of an invitro study, the following conclusions can be drawn:-

Highest shear bond strength values were seen in composites followed by Zirconomer and Nano-hydroxyapitite (GIC). Nano-HAp showed the highest mean micro hardness values when compared to Zirconomer and composites. Nano-HAp can be used as an alternative restorative material.

Tables:-

\begin{tabular}{|l|l|l|l|c|c|}
\hline & (I) Group & Vs & (J) Group & P-Value & Inferences \\
\hline \multirow{3}{*}{$\begin{array}{l}\text { Shear bond } \\
\text { strength }\end{array}$} & Group A & Vs & Group B & 0.000 & S \\
\cline { 2 - 6 } & Group A & Vs & Group C & 0.000 & S \\
\cline { 2 - 6 } & Group B & Vs & Group C & 0.203 & NS \\
\hline
\end{tabular}

Table 1:- Intergroup comparison Shear bond strength values using Post Hoc Tukey’s tests.

\begin{tabular}{|l|l|l|l|c|c|}
\hline & (I) Group & Vs & (J) Group & P-Value & Inferences \\
\hline \multirow{3}{*}{ Micro hardness } & Group A & Vs & Group B & 0.000 & S \\
\cline { 2 - 6 } & Group A & Vs & Group C & 0.000 & S \\
\cline { 2 - 6 } & Group B & Vs & Group C & 0.000 & S \\
\hline
\end{tabular}

Table 2:-Intergroup comparison of Micro hardness values using Post Hoc Tukey's tests.

Figures:-

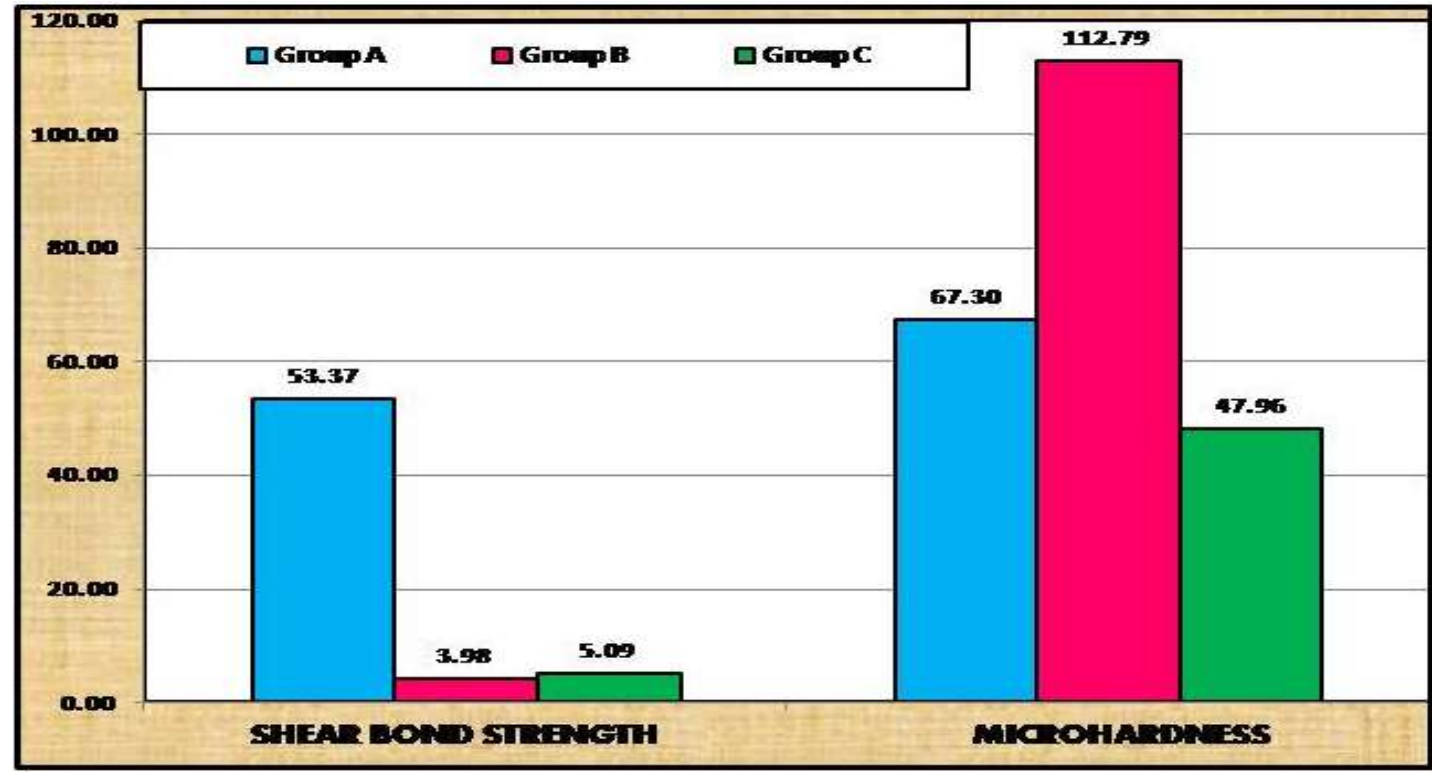

Bar Diagram 1:- Shear bond strength and microhardness values of individual groups.

Source(s) of support: None

Presentation at a meeting: None

Conflicting Interest : None 


\section{References:-}

1. Choudhary K, Nandlal B. Comparative evaluation of shear bond strength of nano-hydroxyapatite incorporated glass ionomer cement and conventional glass ionomer cement on dense synthetic hydroxyapatite disk: An in vitro study. Indian J Dent Res 2015;26:170-5.

2. Sharafeddin F, Azar MR, Feizi N, Salehi R. Evaluation of Surface Microhardness of Silver and Zirconia Reinforced Glass-ionomers with and without Microhydroxyapatite. J Dent Biomater, 2017;4(4):454- 460.

3. Asafarlal S (2017) Comparative Evaluation of Microleakage, Surface Roughness and Hardness of Three Glass Ionomer Cements - Zirconomer, Fujii IX Extra GC and Ketac Molar: An In Vitro Study. Dentistry 7: 427.

4. Gu YW, Yap AUJ, Cheang P, et al. Development of zirconia-glass ionomer cement composites. J non-cryst solids. 2005;351:508-14.

5. Lee JJ, Lee YK, Choi BJ, Lee JH, Choi HJ, Son HK, et al. Physi-cal properties of resin-reinforced glass ionomer cement modified with micro and nano-hydroxyapatite. J Nanosci Nanotechnol. 2010;10:5270-6.

6. Goenka S, Balu R, Sampath Kumar TS. Effects of nanocrystalline calcium deficient hydroxyapatite incorporation in glass ionomer cements. J Mech Behav Biomed Mater. 2012;7:69-76.

7. Rasheed M, Raghad AM. Assessment of diametral tensile strength and microhardness of Glass ionomer reinforced by different amounts of Hydroxyapatite. J Bagh Coll Dentistry. 2006;18:17-20.

8. Arita K, Yamamoto A, Shinonaga Y, et al. Hydroxyapatite particle characteristics influence the enhancement of the mechanical and chemical properties of conventional restorative glassionomer cement. Dent mater J. 2011;30:672-83.

9. Choudhary K, Nandlal B. Comparative evaluation of shear bond strength of nano-hydroxyapatite incorporated glass ionomer cement and conventional glass ionomer cement on dense synthetic hydroxyapatite disk: An in vitro study. Indian J Dent Res 2015;26:170-5.

10. Moshaverinia A, Ansari S, Moshaverinia M, Roohpour N, Darr JA, Rehman I. Effects of incorporation of hydroxyapatite and fluoroapatite nanobioceramics into conventional glass ionomer cements(GIC). Acta Biomater 2008;4:432-40. 